\title{
A Conformational Change of Poly(2-vinylpyridine) in Solution as Revealed by Potentiometric Titration
}

\author{
Tsuneko Kitajima-Yamashita \\ Institute for Chemical Research, Kyoto University, Uji, Kyoto-fu, Japan.
}

(Received March 30, 1972)

\begin{abstract}
The ionization behavior of isotactic and atactic poly(2-vinylpyridine) (P2VP) in water-ethanol mixtures containing dilute hydrochloric acid is investigated by means of potentiometric titration. The degree of ionization of $\mathrm{P} 2 \mathrm{VP}, \beta$, is directly determined by measuring a UV absorbance at $2625 \mathrm{~A}$. Stoichiometric proton concentrations $\left(\mathrm{pH}_{\mathrm{c}}\right)$ are determined by applying Gran's procedure proposed for nonaqueous systems. Potentiometric titration curves established for the polymer species with different steric isomerism indicate that the atactic polymer exists in solution as a stronger base than the isotactic polymer. The electrostatic free energies required to ionize the polymers are calculated to be 2000 and $1800 \mathrm{cal} /$ equiv mol for isotactic and atactic polymer, respectively. A plot of $\mathrm{pH}_{\mathrm{c}}$ vs. $\beta$ constructed with data for the atactic polymer shows a plateau region in a range of $\beta$ between 0.2 and 0.4 , while such a region is not observed for the isotactic polymer. This feature suggests that a conformational transition from one assumed at a low degree of ionization to another assumed at a high degree of ionization occurs in the atactic polymer chain. The free energy change for the conformational transition at the discharged state is estimated to be ca. $300 \mathrm{cal} /$ equiv mol according to the approach proposed by Nagasawa-Holtzer. In connection with the above findings, the viscometric behavior of the polymer species with different steric isomerism at $\beta=0.3$ is also investigated. No significant difference in the hydrodynamic behavior between the isotactic and atactic species is detected.
\end{abstract}

KEY WORDS Polyelectrolyte / Poly(2-vinylpyridine) / Potentiometric Titration / pH Determination / Nonaqueous Solution / Conformational Transition / Electrostatic Free Energy / Retention of Conformation / Intrinsic Viscosity /

A large number of conformational studies on the behavior of tactic polyelectrolytes in solution have been presented in the hope of exploring the following two points; whether any conformational transition in the polymer chain really occurs due to electrostatic forces and other interactions, ${ }^{1-4}$ and whether the higher-order conformation resulting from the stereoregularity of polymer chain is retained even in solution. ${ }^{5-10}$

Anomalies in viscosity and potentiometric titration of polymethacrylic acid (PMA) solution have been observed when the degree of ionization is increased, and interpreted in terms of uncoiling of the polymer chains. ${ }^{11-13}$ Mandel assumed that PMA chains undergo a cooperative transition between two mean conformations or states, namely a compact and expanded conformation at lower and higher ionization degrees, respec- tively. ${ }^{14-16}$ The mechanism of stabilizing the compact conformation at lower ionization degrees has been discussed by different authors in different ways. ${ }^{2,4,15,16}$ In relation to these arguements it seems important to note that both the ionic and hydrophobic groups attached to the main chain are concerned closely with conformational transitions which polyelectrolytes in solution undergo.

We will now consider a tactic polyelectrolyte which carries no hydrophobic group. This. polymer will assume a specific conformation in solid state. When such a polymer is dissolved in a solvent, the question arises whether or not the chain conformation could still be retained in solution, and if retained, whether or not it would be changed with the ionization. degree so that a conformational transition occurs. 
Lifson has expected theoretically that a polyelectrolyte chain would assume a compact conformation when the ionization degree is increased. ${ }^{17}$ On the other hand, Nagasawa, et al., compared potentiometric titration curves obtained for tactic polyacrylic acid (PAA) and also for tactic PMA with those calculated by assuming that the specific conformation expected relevant to each is retained in solution, and found agreement between theory and experiment., ${ }^{5,6}$ This argument for PAA was supported later by the same authors with high-resolution NMR. ${ }^{7}$

2-vinylpyridine can be polymerized to produce isotactic and atactic polymers ${ }^{18,19}(\mathrm{P} 2 \mathrm{VP})$. These polymers with different steric isomerism are soluble not only in water containing hydrochloric acid but also in some organic solvents. When the polymer is dissolved in an aqueous acid solution, protonation occurs and polymers behave as a cationic polyelectrolyte with no hydrophobic group as follows:<smiles>CCC(C)c1[nH]cc(Cl)c1C=[In]</smiles>

This nature of P2VP resembles that of PAA and will be appropriate for generalizing the result that has been obtained for PAA. In addition, this polymer has advantages in determining the true ionization degree ${ }^{20}$ as described later, and also in characterizing the polymer because of its high solubility in organic solvents.

The present work deals with the conformational problem of tactic and atactic P2VP in solution. Several authors ensured ${ }^{5,6,7,21}$ that the potentiometric titration curve of polyelectrolytes solution sensitively reflects changes in the conformation of a polymer chain when such a change takes place in a localized region on the chain. On the other hand, the information drawn from the potentiometric titration curve of polyelectrolytes solution is concerned with the single-ion activity of the counter ion. It seems important to recognize the statement that the single-ion activities cannot be determined without some assumptions and must be regarded as working quantities. Special care is necessary ${ }^{23,24}$ to recognize the inherent uncertainty of any numerical value assigned. Thus our concern is a comparative study on tactic and atactic P2VP in solution with respect to the behavior detected by potentiometric titration. It is shown in this paper that a conformational change is observed not for the isotactic but for the atactic polymer.

\section{EXPERIMENTAL}

\section{Materials}

Samples of isotactic P2VP were prepared according to a procedure proposed by Natta and his colleagues. ${ }^{18}$ Polymerization was made in toluene at $0^{\circ} \mathrm{C}$ with phenylmagnesium bromide as initiator. The product was subjected to extraction with hot acetone for several hours, and a portion insoluble in acetone was isolated and dissolved in monochlorobenzene. The polymer was recovered by pouring this solution into a large volume of $n$-hexane. Dissolving and recovering of the sample were repeated several times by making use of the procedure mentioned above. The sample thus obtained was ascertained to be crystalline by means of X-ray diffraction. A series of isotactic P2VP samples with different molecular weights was prepared by changing the ratio of initiator to monomer, and they are designated I-2, I-6, and I-8. Melting points of the acetone-insoluble portions were in a range between 191 and $197^{\circ} \mathrm{C}$.

Eight samples of atactic P2VP with different molecular weights were prepared at $-78^{\circ} \mathrm{C}$ by an anionic polymerization with sodium-diphenyl complex as initiator in tetrahydrofuran (THF). The product was recovered from the THF solution with $n$-hexane used as precipitant. Molecular weights of the samples were regulated by changing the ratio of initiator to monomer. They are designated A-1, A-2, ., and A-8 (sample A-5 was not used in this study).

\section{Characterization}

Intrinsic-viscosity determination was made with a capillary viscometer of the Ubbelohde suspended-meniscus type at $25^{\circ} \mathrm{C}$ in $N, N$-dimethylformamide (DMF) and also in $0.02-N$ hydrochloric acid solution containing $0.1-M$ sodium chloride. The intrinsic viscosity at $63^{\circ} \mathrm{C}$ in toluene, which is a poor solvent for P2VP, was also determined. Number-average and weight-average molecular weights $\left(M_{n}\right.$ and $M_{w}$, respectively) were determined in DMF by osmotic 


\section{T. KitAJIMA-Yamashita}

pressure measurements and by light scattering, respectively. Osmotic pressure measurements were conducted in DMF at $37^{\circ} \mathrm{C}$ for five atactic samples. A high speed membrane osmometer (Mechlolab Model 502) fitted with an adequately conditioned membrane Ultracellafilter "allerfeinst" (Membranfilter, Göttingen) was used. Light scattering measurements were made with a Shimadzu light scattering photometer equipped with a thermostatted water jacket and a cylindrical cell. Vertically polarized light beams at wave lengths 4360 and $5460 \mathrm{~A}$ were used. Sample solutions were cleaned optically by filtration with an Ultracellafilter "feinst". When the solute was isotactic or of high molecular weights, the optical cleaning was carried out by centrifugation at a rotor speed between 15000 and $20000 \mathrm{rpm}$ for one hour. A preparative centrifuge (Hitachi $55 \mathrm{p}$ ) was employed. For some samples values of $M_{w}$ were determined in ethanol according to the sedimentation equilibrium method using a Hitachi ultracentrifuge (UCA 1-A) furnished with a Schlieren optical system and an eightchannel centerpiece. Each centrifugation run was operated at a rotor speed between 5000 and $10000 \mathrm{rpm}$. The Yphantis procedure ${ }^{25}$ was applied to the analysis of data. Results of the characterization are summarized in Table I. A sample designated S-8 in the table was kindly supplied by Dr. S. Arichi, Faculty of Science, Kumamoto University.

Table I. Results of polymer characterization

\begin{tabular}{|c|c|c|c|c|}
\hline $\begin{array}{c}\text { Sample } \\
\text { code }\end{array}$ & $\begin{array}{c}{[\eta], \mathrm{d} l / \mathrm{g}} \\
(\text { in } \\
\mathrm{DMF})\end{array}$ & $\begin{array}{c}{[\eta], \mathrm{d} l / \mathrm{g}} \\
(\text { in } \\
0.02-N \mathrm{HCl} \\
+0.1-N \\
\mathrm{NaCl})\end{array}$ & $M_{w} \times 10^{-4}$ & $M_{n} \times 10^{-4}$ \\
\hline A-2 & $0.74_{0}$ & $1.74_{5}$ & 21.2 & 8.3 \\
\hline A-1 & $0.56_{6}$ & $2.01_{4}$ & 22.9 & 15.9 \\
\hline A-3 & $0.93_{5}$ & $3.22_{5}$ & 52.5 & 30.2 \\
\hline A-8 & $1.13_{0}$ & $4.62_{0}$ & 67.7 & 49.0 \\
\hline A-7 & $1.60_{0}$ & $5.16_{5}$ & 105.1 & 48.8 \\
\hline A-6 & $2.94_{0}$ & 10.00 & 240.0 & - \\
\hline A-4 & $4.85_{5}$ & - & 540.0 & - \\
\hline $\mathrm{I}-2$ & $0.32_{9}$ & 1.14 & $11.7^{\mathrm{a}}$ & - \\
\hline I-6 & $0.71_{6}$ & 2.60 & $33.0^{\mathrm{a}}$ & - \\
\hline S-8 & $0.82_{0}$ & 3.00 & 43.9 & - \\
\hline $\mathrm{I}-8$ & $0.94_{1}$ & 3.34 & 44.8 & - \\
\hline
\end{tabular}

a Determined by sedimentation equilibrium.

\section{Determination of the Degree of Ionization}

Because of the low basicity of the pyridine groups, the usual procedure to estimate the ionization degree from the added volume of acid solution is insufficient to give the required relationship between the ionization degree $\beta$ and the proton concentration $\mathrm{pH}$. For poly(4-vinylpyridine) in aqueous acid solution it has been found that a UV absorption coefficient at 2625 A characteristic of the pyridine residue is increased with increasing proton concentration in the medium. ${ }^{26}$ This feature may serve as a means for determination of the true ionization degree for P2VP. First we ensured that the absorption coefficient did not change when the hydrochloric acid concentration became higher than $1 N$, while it was kept constant in some alcohols with different dielectric constants. This allowed us to assume that the polymer in $1-N$ hydrochloric acid and in ethanol are in a fully ionized and in a fully discharged state, respectively. In other words, the absorption coefficients obtained in 1-N hydrochloric acid and in ethanol were regarded as those for $\beta=1$ and $\beta=0$, respectively. The absorption coefficients $\varepsilon$, determined at $2625 \mathrm{~A}$ for isotactic and atactic samples in the both states are listed in Table II. It is noted that the value is different for different steric isomerism. Now when a linear relation between $\beta$ and $\varepsilon$ is assumed, any value of $\beta$ between 0 and 1 may be assessed by determining $\varepsilon$. The above assumption was verified by establishing that the Beer law holds for solutions of P2VP with a fixed acid concentration $0.1 \mathrm{~N}$ at least in a polymer concentration range from $(1 \sim 4) \times 10^{-4}$ equiv mol/l. This means, in turn, that the value of $\varepsilon$ is independent of polymer concentration in the range studied so far. The UV absorption measurement was made with a Shimadzu multipurpose recording spectrophotometer (MPS-50L, Shimadzu Seisakusho Ltd., Kyoto).

Table II. Absorption coefficients at $2625 \mathrm{~A}$ characteristic of pyridine residue

\begin{tabular}{ccc}
\hline \multirow{2}{*}{$\begin{array}{c}\text { Sample } \\
\text { code }\end{array}$} & \multicolumn{2}{c}{ Absorption coefficient $(1 /$ equiv mol cm) } \\
\cline { 2 - 3 } & In ethanol & In $2-N$ hydrochloric acid \\
\hline A-8 & 2900 & 5500 \\
I-8 & 2700 & 5350 \\
\hline
\end{tabular}




\section{pH Determination}

Determination of the activity of the individual ionic species is impossible for the thermodynamic reason, ${ }^{22,27}$ and the value of $\mathrm{pH}$ usually obtained by measuring the electromotive force generated in an electrolytic cell should be regarded as a practical measure of the activity, which is acceptable under some assumptions of single-ion activity coefficients. ${ }^{28}$ However, the validity of such assumptions is unjustifiable for a nonaqueous solution of polyelectrolytes with which we are concerned here. For the purpose we write the usual definition of $\mathrm{pH}$ as

$$
\mathrm{pH}=-\log \left[\mathrm{H}^{+}\right]-\log f_{\mathrm{H}^{+}}
$$

where $\left[\mathrm{H}^{+}\right]$and $f_{\mathrm{H}^{+}}$are the stoichiometric proton concentration and the activity coefficient, respectively. Let us now consider an electrolytic cell constructed as follows

Glass electrode|Solution|Reference electrode

The electromotive force generated by such a cell may be given by

$$
\begin{aligned}
E= & E^{0^{\prime}}-\frac{2.303 R T}{F} \log \left[\mathrm{H}^{+}\right] \\
& -\frac{2.303 R T}{F} \log f_{\mathrm{H}^{+}}+E_{J}
\end{aligned}
$$

where $E^{0 \prime}$ is a constant characteristic of the reference and the glass electrode; $E_{\mathrm{J}}$ is a liquid junction potential associated with the cell; $F$ is the Faraday constant.

When a given solution system contains a neutral salt of an amount sufficiently higher than that of protons present, the activity coefficient $f_{\mathrm{H}^{+}}$may reasonably be assumed to be constant and independent of the proton concentration. ${ }^{27}$ Thus eq 3 may be rewritten

$$
E=E^{0}+\frac{2.303 R T}{F} \mathrm{pH}_{\mathrm{c}}
$$

with

$$
E^{0}=E^{0^{\prime}}+E_{\mathrm{J}}-\frac{2.303 R T}{F} \log f_{\mathrm{H}^{+}}
$$

and

$$
\mathrm{pH}_{\mathrm{c}}=-\log \left[\mathrm{H}^{+}\right]
$$

In the above derivation further assumption that
$E_{\mathrm{J}}$ is constant or negligibly small is made, which holds in usual cases under a constant ionic strength. Use of eq 4 allows us to determine values of stoichiometric proton concentrations without any knowledge of the activity coefficient, that is to say, without any reference solution.

Our main subject is to determine the value of $\mathrm{pH}_{\mathrm{c}}$ in mixtures of water and ethanol containing ionized P2VP and sodium chloride with the aid of eq 4 . To do this, the value of $E^{0}$ defined by eq 5 must be first estimated. This can be made according to a treatment of acidalkali titration data proposed by Gran, ${ }^{29}$ which yields $E^{0}$ as the slope of a plot given by

$$
\begin{aligned}
& \left(V_{0}+V_{\mathrm{B}}\right) 10^{-0.434 F E / R T} \\
& \quad=10^{-0.434 F E^{0} / R T}\left(C_{\mathrm{H}} V_{0}-C_{\mathrm{B}} V_{\mathrm{B}}-\beta C_{\mathrm{p}} V_{0}\right)
\end{aligned}
$$

where $V_{0}$ and $V_{\mathrm{B}}$ are the initial volume of acid solution and the added volume of base solution; $C_{\mathrm{H}}, C_{\mathrm{p}}$ and $C_{\mathrm{B}}$ are the initial concentration of acid, polyelectrolyte, and base solution, respectively.

In practice, a water-ethanol mixture $(1: 1$ by volume) acidified with a small amount of hydrochloric acid (e.g., $5 \times 10^{-3} N$ ), which further contains a small amount of P2VP $\left(1.93 \times 10^{-4}\right.$ equiv $\mathrm{mol} / \mathrm{l}$ ) and a moderately large amount of sodium chloride $(0.1 M)$, was prepared. To weigh the small amount of polymer sample, a microbalance with a precision of $\pm 1 \mu \mathrm{g}$ was used. This solution was placed in a titration apparatus constructed by combining a $\mathrm{pH}$-meter (42 A, Yanagimoto MFG. Co., Ltd., Kyoto) with a glass electrode (MG11C, Yanagimoto, MFG. Co., Ltd., Kyoto) and a reference calomel electrode having a double salt bridge (Double Junction 2535-05T, Horiba Instruments Inc., Kyoto). The pH-meter has a sensitivity of \pm 0.05 -pH unit. The electrodes were set inside the solution throughout the titration, since the value of $E^{0^{\prime}}$ and $E_{J}$ might be changed when the electrode is brought out of the solution. Then the titration was made at $14 \pm 1^{\circ} \mathrm{C}$ with caustic soda $(0.1346 N)$ dissolved in a waterethanol mixture with the same solvent composition and salt concentration as those in the test solution. Electromotive forces $E$ thus generated at various stages of $V_{\mathrm{B}}$ were determined and plotted against the value of $V_{B}$ in accordance with eq 7. Figure 1 shows the Gran plot. 


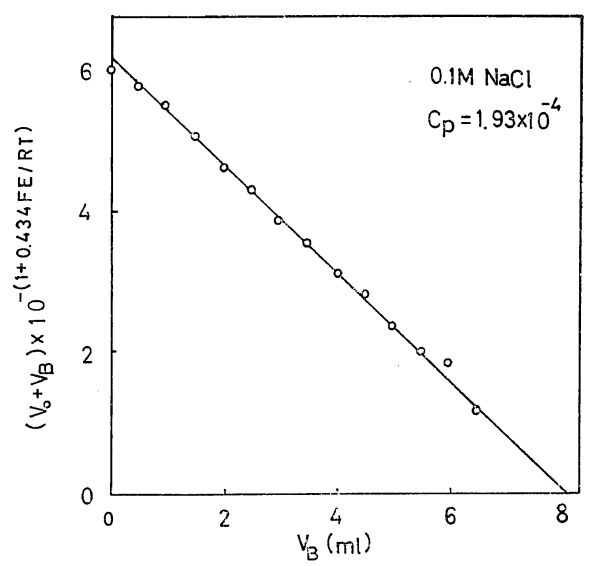

Figure 1. Gran's plot for sample A-8 in (1:1)water ethanol mixture containing $0.1-M \mathrm{NaCl}$ in the presence of polymer at concentration $1.93 \times$ $10^{-4}$ equiv $\mathrm{mol} / l$.

The constancy of $E^{0}$ for this system may reasonably be assumed by good linearity of the plot indicated in the figure. The value of $V_{\mathrm{B}}$ at which the left hand side of eq 7 is extraporated to zero, is designated as $V_{\mathrm{e}}$. The concentration of proton, $\left[\mathrm{H}^{+}\right]$is calculated as $C_{\mathrm{B}}\left(V_{\mathrm{e}}-V_{\mathrm{B}}\right) /$ $\left(V_{0}+V_{\mathrm{B}}\right)$. The value of $E^{0}$ is determined from the difference between $\mathrm{pH}_{\mathrm{r}}$ end $\mathrm{pH}_{\mathrm{c}}$, where $\mathrm{pH}_{\mathrm{r}}$ is defined as $0.434 F E / R T$.

The value of $V_{\mathrm{e}}$ may be regarded as constant for this system as shown in the plot of Figure 1 , and this means that the change of $\beta$ during the addition of base solution may be neglected in the limit of experimental error. In fact, the change of $V_{e}$ owing to the change of $\beta$ is estimated with values of $C_{\mathrm{p}}\left(10^{-4}\right), C_{\mathrm{H}}\left(10^{-3}\right)$, and $\beta(0.01 \sim 0.015)$, and proved to be of the order of $10^{-3}(\%)$.

Then to the above test system, for which $E^{0}$ has been determined, moderately concentrated hydrochloric acid was added in such a way that no changes in the solvent composition and salt concentration take place. The electromotive force $E$ was read off at each addition of acid solution, and values of $\mathrm{pH}_{\mathrm{c}}$ for this system were evalvated by using eq 4 with the value of $E^{0}$ given previously. At each stage of $\mathrm{pH}_{\mathrm{c}}$ value a constant amount of the solution $(5 \mathrm{ml})$ was pipetted out and subjected to the UV absorption measurement in order to determine the ionization degree $\beta$ as a function of $\mathrm{pH}_{\mathrm{c}}$. The volumetric correction for the polymer concentration was carried out. This procedure was applied to the establishment of relation between $\mathrm{pH}_{\mathrm{c}}$ and $\beta$ for the isotactic and atactic samples, I-8 and A-8, respectively.

\section{RESULTS}

As mentioned in the last section our main concern was to determine the values of $\mathrm{pH}_{\mathrm{c}}$ at the different degree of ionization $\beta$. Before we construct relations between $\mathrm{pH}_{\mathrm{c}}$ and $\beta$, we think it appropriate to make some comments on the validity of eq 4 with which $\mathrm{pH}_{\mathrm{c}}$ was evaluated. During the $\mathrm{pH}_{\mathrm{c}}$ determination, hydrochloric acid was added to the test solution. This will accompany some change in the ionic strength of the whole system through changes in the degree of ionization and polymer concentration. If the extraneous salt is present at concentration comparable to that of polyion, the change in polyion concentration will influence the proton activity so that the constancy of $E^{0}$ does no more hold and eq 4 becomes invalid. To avoid such a difficulty, we worked at enough high concentrations of extraneous salt $\left(10^{-1} \mathrm{~mol} / l\right)$. Further to ensure this point experimentally, we applied the Gran procedure to solutions containing different amounts of polymer ranging from 0 to $4 \times 10^{-3}$ equiv $\mathrm{mol} / l$ under a fixed salt concentration $(0.1 M)$. Figure 2 shows the result, which is given by plotting values of $E^{0}$ expressed in $\mathrm{pH}$ units against polymer concentrations. A slight dependence of $E^{0}$ on polymer concentration is seen, but $E^{0}$ may be regarded as constant in the polymer concentration range studied, if one takes into account that the precision limit of the measurement is within $c a$. \pm 0.05 in $\mathrm{pH}$ units.

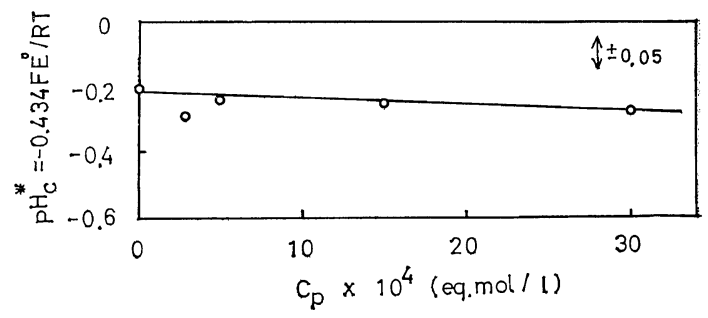

Figure 2. Effect of polymer concentration on $\mathrm{pH}_{\mathrm{c}}$ * for P2VP solution, where $\mathrm{pH}_{\mathrm{c}}{ }^{*}=-0.434 E^{\circ} F / R T$. For the details, see text. 
As mentioned in the last section we determined the $\mathrm{pH}_{\mathrm{c}}$ values by potentiometric titration at each degree of ionization $\beta$ deduced from the UV absorption. Thus titration curves can be constructed by plotting the $\mathrm{pH}_{\mathrm{c}}$ value against $\beta$. The titration curves obtained for isotactic and atactic samples, I-8 and A-8, are shown in Figure 3. Inspection of the figure reveals that the degree of ionization of I-8 is always smaller than that of A-8 compared at the same $\mathrm{pH}_{\mathrm{c}}$ value. This means that atactic polymer is a stronger base than isotactic polymer.

A discontinuity in the plot for A-8 which may be observed at between 0.2 and 0.4 of $\beta$ is absent in the plot for I-8. This feature of the titration curve of A-8 is also seen in the HendersonHasselbach plot $^{30}$ in Figure 4. For A-8 two distinctly linear regions are seen when the $\mathrm{pH}_{\mathrm{c}}$ value is plotted against $\log (\beta / 1-\beta)$. If the titration curve is interpreted in terms of a conformational transition between two conformations, each linear part of the titration curve of A-8 corresponds to a region where one of the two conformations is predominantly present and the region held between the two linear lines may

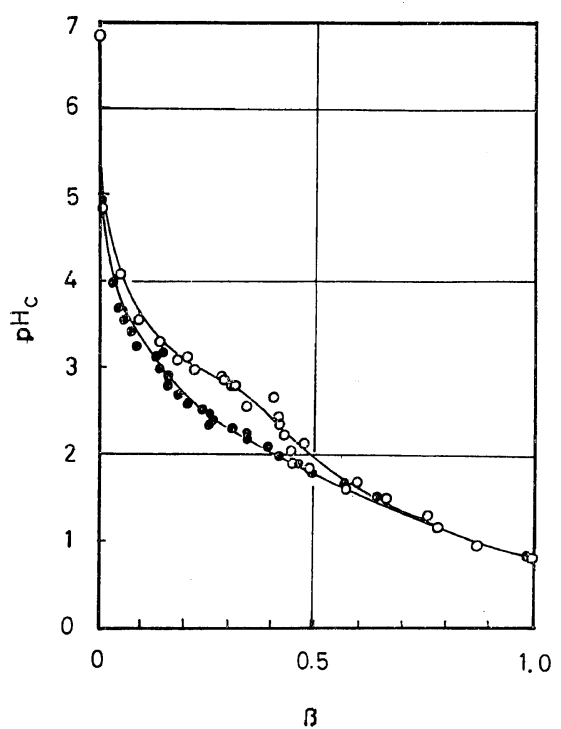

Figure 3. Potentiometric titration curves obtained for isotactic and atactic P2VP (I-8, A-8) in water -ethanol mixture containing $0.1-M \mathrm{NaCl}$ at $14 \pm$ $1^{\circ} \mathrm{C}$. Symbols (-) and (O) are referred to I-8 and $\mathrm{A}-8$, respectively.

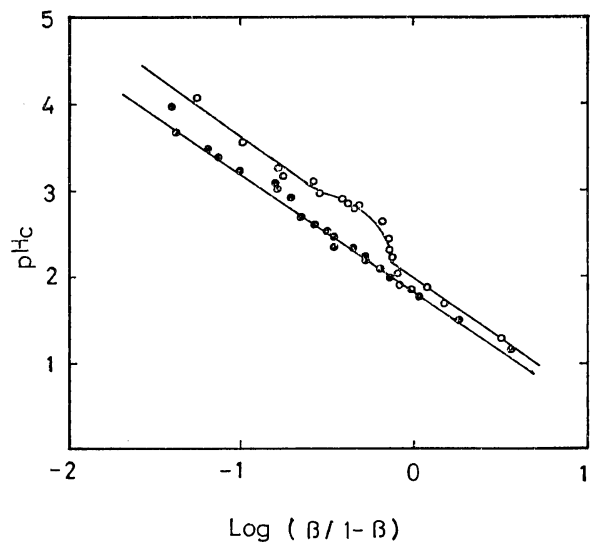

Figure 4. Henderson-Hasselbach plots constructed for I-8 (०) and A-8 (O).

corresponds to a region where the conformational transition occurs.

\section{DISCUSSION}

A conformational transition between chain forms I and II at lower and higher degrees of ionization, respectively, was observed for atactic P2VP in solution. The free-energy change associated with this transition occurring at the discharged state, $\Delta F^{0}$, is given by

$$
\frac{C_{\mathrm{II}}^{0}}{C_{\mathrm{I}}{ }^{0}}=\exp \left(-\Delta F^{0} / R T\right)
$$

where $C_{\mathrm{I}}^{0}$ and $C_{\mathrm{II}}^{0}$ are the concentrations of form I and II at discharged state, respectively. Thus $\Delta F^{0}$ may be estimated if a ratio $C_{\mathrm{II}}^{0} / C_{\mathrm{I}}^{0}$ is known. On the basis of a theory for protolytic equilibria of polyvalent bases carrying $z$ charges, ${ }^{32}$ Nagasawa and Holtzer have derived an expression for this ratio, ${ }^{31}$ which leads $\Delta F^{0}$ to

$$
\begin{aligned}
\frac{\Delta F^{0}}{N}= & -2.303 R T\left[\int_{\mathrm{pH}(z=0)}^{\mathrm{pH}}\left(\beta_{\mathrm{I}}-\beta_{\mathrm{II}}\right) \mathrm{d} p H\right. \\
& \left.+\frac{1}{N} \log \frac{C_{\mathrm{II}}}{C_{\mathrm{I}}}\right]
\end{aligned}
$$

where $C_{\mathrm{I}}$ and $C_{\mathrm{II}}$ are the total concentrations of form I and II at their all possible charged states including the discharged state, respectively, and $\beta_{I}$ and $\beta_{I I}$ are the degree of ionization of polyion species with each chain form, and $N$ is the degree of polymerization. 


\section{T. KitAJima-YamashitA}

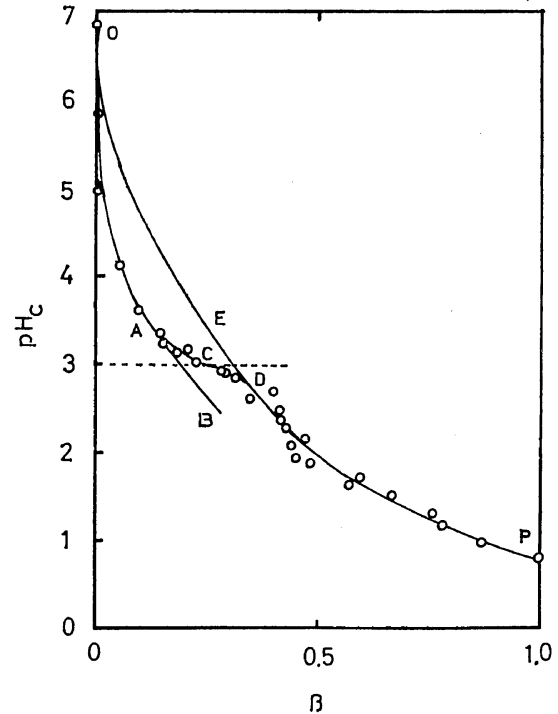

Figure 5. Potentiometric titration curves represented in the form of $\mathrm{pH}_{\mathrm{c}} v s . \beta$ plot. Curves $\mathrm{AB}$ and $O D$ are hypothetical titration curves for species of form I and form II, respectively. Curve ACD is the actual curve in the transition region and at point $\mathrm{C}$ the concentrations of species of forms $\mathrm{I}$ and II are equal to each other.

When one finds out a $\mathrm{pH}_{\mathrm{c}}$ value, $\mathrm{pH}_{1}$, at which $C_{\mathrm{I}}$ becomes identical with $C_{\mathrm{II}}$, the integration involved in eq 10 may simply be reduced to

$$
\frac{\Delta F^{0}}{N}=-2.303 R T \int_{\mathrm{pH}(z=0)}^{\mathrm{pH}_{1}}\left(\beta_{\mathrm{I}}-\beta_{\mathrm{II}}\right) \mathrm{d} p H
$$

To estimate $\Delta F^{0}$ from our experimental results, the values of $\mathrm{pH}_{\mathrm{c}}$ are plotted against $\beta$ in Figure 5. Taking into consideration that the conformational transition occurs, we extrapolate the data points appearing in lower $\beta$ from $\beta=0$ to higher $\beta$ to obtain a cuvre $\mathrm{AB}$ in the figure, while we extrapolate those in higher $\beta$ from $\beta=1$ to lower $\beta$ to obtain a curve DEO. The former and latter curves may correspond to hypothetical titration curves that would be observed for forms I and II, respectively. Let us denote the degree of ionization for any data point existing between the curves (in the transition region) with $\beta_{\mathrm{a}}$. This value may be regarded as that for a mixture of species of forms I and II, and will be given by

$$
\beta_{\mathrm{a}}=x \beta_{\mathrm{I}}+(1-x) \beta_{\mathrm{II}}
$$

where $x$ is the fraction of species of form $\mathrm{I}$. The first task required by eq 11 is to find out values of $\beta_{a}$, which correspond to $x=0.5$ at different $\mathrm{pH}_{\mathrm{c}}$, by using the hypothetical titration curves. A locus of values of $\beta_{a}$ is drawn and a cross point between the locus and the observed titration curve designated AD in Figure 5 is easily found. The $\mathrm{pH}_{\mathrm{c}}$ value at this cross point will be $\mathrm{pH}_{1}$ so far as the above hypothesis is valid. With this procedure $\mathrm{pH}_{1}$ was determined 3.0. Graphical integration according to eq 11 was then carried out, which yielded 300 cal/equiv mol for $\Delta F^{0} / N$. Estimation of $\Delta F^{0}$ according to the theory of Zimm and Rice ${ }^{33}$ was not made, since the plot between $\left[\mathrm{pH}_{\mathrm{c}}+\log (\beta / 1\right.$ $-\beta)]$ and $\beta$ indicated poor linearity, differing from those obtained for some synthetic polypeptides. $^{34,35}$

The abrupt change in the titration curves as revealed for atactic P2VP has already been found for PMA. ${ }^{1-4}$ The hydrophobic methyl groups in the PMA chain may play an important role in retaining the compact formation at the lower degree of ionization. In the P2VP chain it is not probable, however, that the pyridine residues behave similar to the methyl groups in PMA. The value of free-energy change for the conformational transition in atactic P2VP is about $300 \mathrm{cal} /$ equiv mol, and this value is of the same order as those found by the similar analysis of titration data for poly(L-lysine) in aqueous salt solution $(100-200 \mathrm{cal} /$ equiv mol $),{ }^{34}$ poly(Lglutamic acid) in water $(150-300 \mathrm{cal} /$ equiv mol), ${ }^{35}$ and PMA in aqueous solution (100-250 cal/equiv mol. ${ }^{4,14,36}$ The question why the conformational trasition occurs not for isotactic but atactic P2VP is still open to discussion.

The electrostatic free energy at $\beta=1$ of isotactic and atactic P2VP may be obtained by graphic integration according to the equation ${ }^{37}$

$$
F^{\mathrm{e}}(\beta)=\int_{0}^{\beta}\left[-\mathrm{pK}_{0}-\mathrm{pH}_{\mathrm{c}}-\log (\beta / 1-\beta)\right] \mathrm{d} \beta,
$$

where $\mathrm{pK}_{0}$ is the intrinsic ionization exponent. To estimate the free energy, values of $\left[\mathrm{pH}_{\mathrm{c}}+\right.$ $\log (\beta / 1-\beta)]$ were plotted against $\beta$. These plots for the isotactic and atactic polymer are shown in Figure 6. Values of $F^{11}$ were evaluated 2000 and $1800 \mathrm{cal} /$ equiv mol for isotactic and atactic P2VP, respectively. In general the 
A Conformational Change of Poly(2-vinylpyridine)

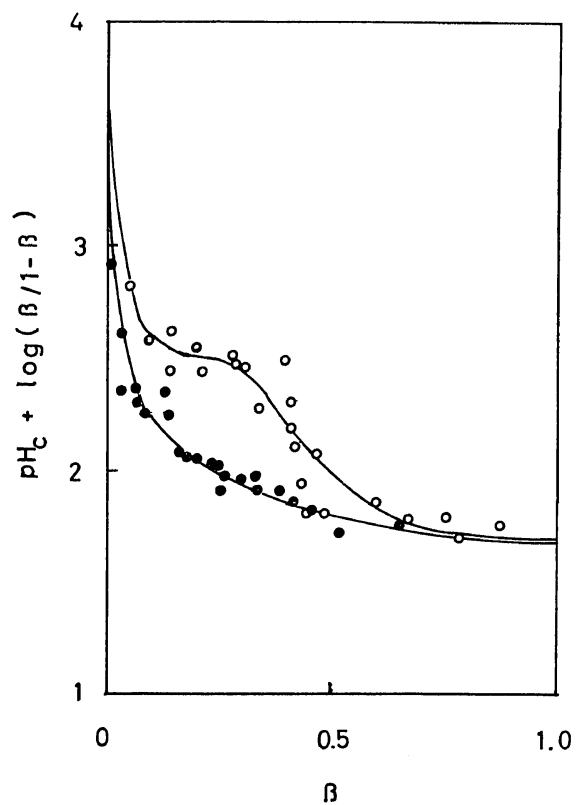

Figure 6. Plots of $\mathrm{pH}_{\mathrm{c}}+\log (\beta / 1-\beta)$ against $\beta$. Symbol (O) and $(\bigcirc)$ are priferred to I-8 and A-8, respectively.

value of $F^{\mathrm{e}}$ is sensitively affected by the choice of $\mathrm{pK}_{0}$. However it is obvious that the order of magnitudes of $F^{\mathrm{el}}$ obtained for the both P2VP will have nothing to do with the $\mathrm{pK}_{0}$ value. The difference in $F^{\mathrm{e} 1}$ may imply that the spatial distribution of pyridine residues of the isotactic chain differs from that of the atactic, and, therefore, may be attributed to the difference in the conformations assumed by these polymers in solid state. ${ }^{18}$ At the same time this result permits us to presume that the chain conformation of tactic polymers could still be retained in dilute solution. If such a postulation be permissible, the isotactic chain will have a charge density higher than that of the atactic so that the difference in $F^{\mathrm{e} 1}$ is understandable. In fact, a theoretical treatment along the analogous lines to the above has been presented with success by Nagasawa, et al., for PMA and PAA. ${ }^{5}$

Further to prove the posturation given above, we have determined intrinsic viscosities of the isotactic and atactic samples in $0.02 \mathrm{~N}$ hydrochloric acid solution containing $0.1-M$ sodium chloride, in which the degree of ionization is

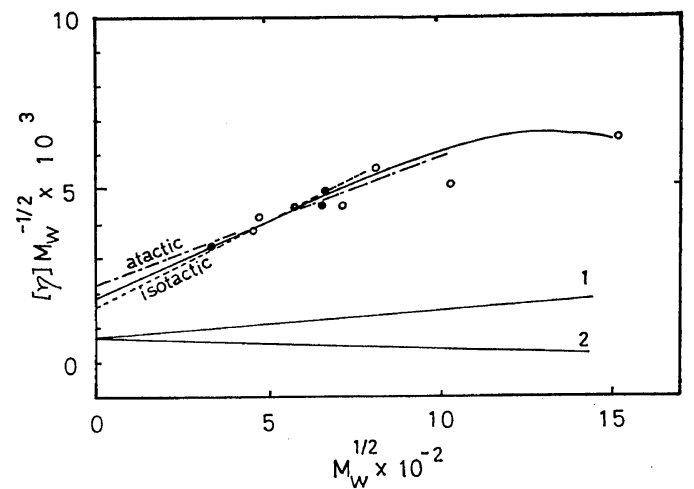

Figure 7. Stockmayer-Fixman's plots for isotactic and atactic $\mathrm{P} 2 \mathrm{VP}$ in $0.02-N$ hydrochloric acid with $0.1-M \mathrm{NaCl}$ at $25^{\circ} \mathrm{C}$. Symbols (O) and (O) are data points for isotactic and atactic P2VP, respectively. Curves 1 and 2 indicate the plot obtained for atactic P2VP in DMF at $25^{\circ} \mathrm{C}$ and in toluene at $63^{\circ} \mathrm{C}$, respectively. Curves (---) and (-.--) are tentatively drawn for isotactic and atactic polymer, respectively.

fixed at $c a$. 0.3. The data points were plotted according to the Stockmayer-Fixman equation ${ }^{38}$

$$
\frac{[\eta]}{M^{1 / 2}}=K+0.51 \Phi_{0} B M^{1 / 2}
$$

where $M$ is the molecular weight, $\Phi_{0}$ is the viscosity constant at $\Theta$ point, and $K$ and $B$ are parameters related to short-range and long-range interaction between segments, respectively. The plots are shown in Figure 7, in which data obtained in DMF and toluene are also given for comparison. It is seen that no definite difference in the hydrodynamic behavior is found. The unperturbed dimensions obtained from intercepts on ordinate at $M=0$ are indifferent for both samples. However, if the data points for higher molecular weights are neglected, the intercept for isotactic samples is only slightly smaller than that for the atactic. This result should be compared to the finding of Loucheux, et al. ${ }^{9,10}$ such that isotactic P2VP has a smaller unperturbed dimension than that of the atactic. They interpreted this difference by presuming that a helicoidal structure of the isotactic chain is retained in solution. For the reason aforementioned we also believe that the isotactic polymer may retain some ordered chain structure in solution such as a helicoidal one. However, the difference in 


\section{T. KitAJima-Yamashita}

the unperturbed dimensions is not so definite that one can conclude that the isotactic polymer assumes helicoidal structure. The retention of conformation that the isotactic polymer assumes helicoidal structure. The retention of conformation that we figure out may be concerned with some localized parts of the whole chain so that no pronounced change in the hydrodynamic behavior is expected. Thus the discrepancy between the conclusion of Loucheux, et al., and ours may not be of serious nature.

Acknowledgement. The author is pleased to thank Professor H. Inagaki for his advice, interest, encouragement during this study, Dr. S. Arichi of Kumamoto University for kindly offering the sample and helpful discussions, and Dr. N. Imai of Nagoya University for his helpful suggestions.

\section{REFERENCES}

1. M. Mandel and J. C. Leyte, J. Polym. Sci.3 56, 23 (1962).

2. A. M. Liquori, G. Barone, V. Crescenzi, F. Quagrifoglio, and V. Vitagliano, J. Macromol. Chem., 1, 291 (1966).

3. A. R. Mathieson and J. V. Mclaren, J. Polym. Sci., Part A, 3, 2555 (1965).

4. T. N. Nekrasova, A. G. Gabrielyan, and O. B. Pritsyn, Vyskomol. Soyedin., Ser. A, 10, 297 (1968).

5. M. Nagasawa, T. Murase, and K. Kondo, $J$. Phys. Chem., 69, 4005 (1965).

6. Y. Muroga, K. Kawaguchi, I. Noda, and M. Nagasawa, Preprints, SPSJ 17th Symposium on Macromolecules, Ehime, 1968, Part 2, p. 591,

7. Y. Muroga, I. Noda, and M. Nagasawa, $J$. Phys. Chem., 73, 667 (1969).

8. D. O. Jordan, T. Kurucsev, and M. L. Martin, Trans. Faraday Soc., 65, 598 (1969); ibid., 65, 606 (1969).

9. C. Loucheux and Z. Czlonkowska, J. Polym. Sci., Part C, No. 16, 4001 (1968).

10. C. Loucheux and M. Rinfret, J. Chim. Phys., 65, 235 (1968).

11. R. Arnold and J. Th. G. Overbeek, Rec. Trav. Chim., 69, 192 (1950).

12. A. Katchalsky and H. Eisenberg, J. Polym. Sci., 6, 145 (1951).
13. A. Katchalsky, ibid., 7, 393 (1951).

14. J. C. Leyte and M. Mandel, ibid., Part A, 2, 1879 (1964).

15. M. Mandel and M. G. Standhouder, Macromol. Chem., 80, 141 (1964).

16. M. Mandel, J. C. Leyte, and M. G. Stadhouder, J. Phys. Chem., 71, 603 (1967).

17. S. Lifson, J. Chem. Phys, 29, 89 (1958).

18. G. Natta, G. Mazzanti, P. Longi, G. Dallasta, and F. Bernardini, J. Polym. Sci., 51, 487 (1961).

19. M. Fontanille and P. Sigwalt, Bull. Soc. Chim. France, 11, 4083 (1967).

20. E. B. Hughes, H. H. G. Jellinek, and B. H. Ambrose, J. Phys. Colloid Chem., 53, 410 (1949).

21. L. Kotin and M. Nagasawa, J. Chem. Phys., 36, 873 (1962).

22. E. A. Guggenheim, J. Phys. Chem., 33, 842 (1929).

23. R. G. Bates, "Determination of pH, Theory and Practice," John Wiley, New York, N.Y., 1964, Chapter 3.

24. N. Ise and T. Okubo, J. Phys. Chem., 70, 3025 (1966).

25. D. A. Yphantis, Biochemistry, 3, 297 (1964).

26. A. Katchalsky and I. R. Miller, J. Polym. Sci., 13, 57 (1954).

27. H. S. Harned and B. B. Owen, "The Physical Chemistry of Electrolytic Solution," Reinhold Pub. Corp., New York, N. Y., 1950.

28. G. Scatchard, J. Amer. Chem. Soc., 47, 696 (1925).

29. G. Gran, Analyst, 77, 661 (1952).

30. A. Katchaisky and J. P. Spitnik, J. Polym. Sci., 2, 432 (1947).

31. M. Nagasawa and A. Holtzer, J. Amer. Chem. Soc. 86, 538 (1964).

32. J. T. Edsall and J. Wyman, "Biophysical Chemistry," Academic Press Inc., New York, N. Y., 1958.

33. B. H. Zimm and S. Rice, Mol. Phys., 3, 391 (1960).

34. J. Hermans, Jr., J. Phys. Chem., 70, 510 (1966).

35. J. Hermans, Jr., J. Amer. Chem. Soc., 88, 2418 (1966).

36. T. N. Nekrasova, Ye. V. Anufriyeva, A. M. Yel'yashevich, and O.B. Ptitsyn, Vysokomol. Soyedin., 7, 913 (1965).

37. A. Katchalsky and J. Gills, Rec. Trav. Chem., 68, 879 (1949).

38. W. H. Stockmayer and M. Fixman, J. Polym. Sci., Part C, 1, 137 (1963). 